\title{
Expression analysis of anthocyanin gene induced under phosphorus starvation in maize genotypes with contrasting phosphorus use efficiency
}

\author{
M.J.V. Vasconcelos ${ }^{1}$, J.E.F. Figueiredo ${ }^{2}$ and K.G. Raghothama ${ }^{3}$ \\ ${ }^{1}$ Núcleo de Biologia Aplicada, Embrapa Milho e Sorgo, Sete Lagoas, MG, \\ Brasil \\ ${ }^{2}$ Laboratório de Bioquímica Molecular, Embrapa Milho e Sorgo, Sete \\ Lagoas, MG, Brasil \\ ${ }^{3}$ Department of Horticulture, Purdue University, West Lafayette, IN, USA \\ Corresponding author: J.E.F. Figueiredo \\ E-mail: jose.edson@embrapa.br
}

Genet. Mol. Res. 17 (4): gmr18036

Received June 25, 2018

Accepted August 07, 2018

Published October 16, 2018

DOI http://dx.doi.org/10.4238/gmr18036

\begin{abstract}
Phosphate (Pi) unavailability is a growth-limiting factor for plants. Under Pi-limited conditions, plants activate molecular mechanisms for better acquisition and utilization of this nutrient. In maize, changes in the expression pattern of several $\mathrm{Pi}$ starvation-induced genes, including the $A l$ coding for dihydroflavonol 4-reductase (DFR) involved in anthocyanin biosynthesis, were identified through microarray analysis. In order to elucidate the molecular determinants with a potential role in P use efficiency, we carried out a study on gene expression analysis of the Al phosphate responsive gene by northern blot analysis of total RNA from maize genotypes contrasting for Pi efficiency. Two Pi-efficient (L-03 and L-161-1) and five inefficient (L-11, L-16, L-22, L-53, and L-5046) genotypes of maize were grown for 15 days in hydroponic culture in the presence $(250 \mu \mathrm{M} \mathrm{Pi})$ or absence $(0 \mu \mathrm{M} \mathrm{Pi})$ of phosphate. All genotypes showed an increase in anthocyanin accumulation in roots in the absence of $\mathrm{Pi}(0 \mu \mathrm{M} \mathrm{Pi})$. The Pi-efficient genotype L-36 and the Pi-inefficient genotypes L-16, L22, and L5046 showed the highest levels of anthocyanin accumulation. The Al gene exhibits temporal and spatial expression patterns associated
\end{abstract}


with Pi deficiency. Although there were differences in the expression profile of $\mathrm{Pi}$ starvation induced genes, no consistent expression patterns could be associated with either Pi-efficient or Pi-inefficient genotypes. It appears that Pi efficiency in tropical maize is a complex trait mediated by a coordinated action of genes that are either induced or suppressed in response to Pi-deficiency.

Key words: Acid soil; anthocyanin; abiotic stress; gene expression; northern blot

\section{INTRODUCTION}

The phosphate ion (Pi) is the main form of phosphorus available to plants. Although the Pi content in the soil frequently exceeds the required amount necessary for plant development, this element remains a limiting nutrient for plant growth (Schachtman et al., 1998). This is due to the chemical properties of phosphate, including slow rate of diffusion, formation of insoluble complexes with cations, especially $\mathrm{Ca}$ in alkaline soils and $\mathrm{Al}$ and $\mathrm{Fe}$ in the acidic conditions of tropical soils (Edwards et al., 2016). Thus, much of the mineral $\mathrm{Pi}$ in fertilizers applied to agricultural soils (around 80\%) is unavailable to plants (Fageria et al., 2014).

Plant development is a dynamic and complex process that is often subject to environmental stresses (Jain et al., 2007). In response to these factors, plants have developed many morphological, physiological, biochemical and molecular strategies to adapt their growth to phosphate-limiting conditions (Raghothama, 1999; Jain et al., 2007). Plant tolerance to Pi deficiency can be achieved by increasing the Pi uptake efficiency through the induction of high-affinity Pi transporters (Mehra et al., 2015). Tolerance can also result from active mechanisms used by plants to recover poorly soluble Pi bound to the soil particles by excreting organic acids, phosphatases and RNases for Pi solubilization (Poirier and Bucher, 2002). Under low phosphorus conditions, plants undergo an array of morpho-physiological adaptive changes to increase the efficiency of $\mathrm{P}$ uptake by the roots, and improve Pi availability for the cells (Hasan et al., 2016). Root hair elongation, anthocyanin accumulation, activation of acid phosphatase, and reduced weight are typical plant responses to phosphate starvation (Raghothama, 2000). These alterations indicate that many genes are involved in the adaptation process (Raghothama, 1999; Raghothama and Karthikeyan 2005). Some genes are directly involved in Pi acquisition, transfer, and signal transduction during Pi stress (phosphate transporters, phosphatases), recycling (RNAse, phosphatases) or regulating the transcription of other genes (Młodzińska and Zboińska, 2016, Puga et al., 2017). However, the details of the regulatory mechanisms of gene expression in response to the phosphorus status of a plant are still unclear.

The identification of phosphate stress-responsive genes is crucial for further characterization of the primary mechanisms involved in Pi acquisition and Pi use efficiency in maize. Many studies have been carried out to understand the gene regulatory network involved in Pi uptake by plants (Muchhal and Raghothama, 1999; Zhang et al., 2014; Gu et al., 2016).

Significant accumulation of anthocyanin in leaves is often related to a number of environmental stresses such as phosphorus deficiency (Chalker-Scott 1999; Glover and 
Martin, 2012). Anthocyanins are water-soluble vacuolar pigments abundant in juvenile and senescing plants, and are responsible for most red, blue, and purple colors in higher plants (Glover and Martin, 2012). Anthocyanin production in maize requires the expression of the $A 1, A 2, C 1$, or $P I$ and $R$, or $B z$ regulatory genes (Styles and Ceska, 1975). The enzyme DFR involved in the two branches of the flavonoid pathway converts dihydroflavonol to leucoanthocyanidin (flavan-3,4-diols), which is metabolized to 3-hidroxyanthocyanidins by leucoanthocyanidin dioxygenase (Styles and Ceska, 1975). In maize and others grasses, another flavonoid biosynthetic pathway converts dihydroflavonol to 3-deoxy flavonoids, such as the phlobaphene pigments, which are almost exclusively accumulated in kernel pericarp, silks and cobs (Carletti et al., 2014). In this study, we address the response of the Al gene in seedlings of contrasting maize genotypes developed for phosphorus use efficiency grown hydroponically under different phosphate concentrations.

\section{MATERIAL AND METHODS}

\section{Plant materials}

The genotypes of Zea mays used in this study were developed at Embrapa Maize and Sorghum, by conventional breeding for Pi acquisition efficiency and Pi utilization efficiency under phosphate fertilization (Table 1). The genotypes were grown in dark red oxisols with low and high levels of phosphorus fertilization $(2 \mathrm{mg} / \mathrm{kg}$ and $15 \mathrm{mg} / \mathrm{kg}$, respectively).

Table 1. Pi-efficient and Pi-inefficient genotypes of maize categorized as phosphorus efficient (E and $\mathrm{HE}$ ) and inefficient (I); responsive (R and HR) and non-responsive (NR).

\begin{tabular}{cc}
\hline Maize genotypes & Responsiveness to Pi \\
\hline L-03 & Efficient - HE / HR \\
L-11 & Inefficient - I / IR \\
L-16 & Inefficient -IN / HR \\
L-22 & Inefficient - I / NR \\
L-36 & Efficient - E / HR \\
L- 53 (723) & Inefficient - IN / NR \\
L-161-1 & Efficient - E / R \\
L-5046 & Inefficient - I / NR \\
\hline
\end{tabular}

\section{Plant culture conditions}

Seeds of maize genotypes were germinated in seedling trays containing Scott's ready earth plug mix (Scotts Co., Marysville, $\mathrm{OH}$ ) and grown in the greenhouse for one week. Afterwards, the seedlings were gently washed with tap water to remove the soil medium from the roots, and transferred to one-half-strength modified Hoagland's nutrient solution. After one week, the plants were transferred to fresh Hoagland nutrition solution containing different $\mu \mathrm{M}$ concentrations of $\mathrm{Pi}(0,5,10,25,50,100$, and 250). During the experiments, nutrient solution was replaced every other day. After 15 days treatment, the roots of plants grown under different Pi concentrations were harvested, frozen in liquid nitrogen and RNA was extracted for Northern blot analysis. Since the evaluation of the expression of different phosphate transporters in maize revealed induction at $0 \mu \mathrm{M} \mathrm{Pi}$ and complete suppression at $250 \mu \mathrm{M} \mathrm{Pi}$, these two concentrations were treated as $\mathrm{Pi}$ - and $\mathrm{Pi}+$, respectively for studying the different aspects of Pi deficiency on the expression of the gene Al of maize. For a time-course study, roots from both Pi+ and Pi- treatments were harvested 
sequentially after $1,3,5,6,7,8,12$, and 15 days of growth. Furthermore, after 15 days of growth under $\mathrm{P}+$ and $\mathrm{P}$ - conditions, roots, stems, young leaves and old leaves were harvested separately for evaluating the spatial expression of Pi-induced genes in these tissues.

\section{Measurements of anthocyanin production}

A simple and rapid method was used for the quantification of total anthocyanin content in roots, as described by Abdel-Aal and Hucl (1999). Fresh root samples from both treatments $(\mathrm{Pi}-$ and $\mathrm{Pi}+)$ were ground separately in liquid nitrogen with a mortar and pestle. Approximately one gram of the powdered tissue was suspended with $10 \mathrm{~mL}$ acidified ethanol prepared by mixing $85 \mathrm{~mL}$ of $100 \%$ ethanol and $15 \mathrm{~mL}$ of $1.0 \mathrm{~N} \mathrm{HCl}$, and the $\mathrm{pH}$ was adjusted to 1.0 with $4 \mathrm{~N} \mathrm{HCl}$. The samples in acidified ethanol were mixed, and then centrifuged at $10,000 \mathrm{rpm}$ for 5 minutes at room temperature. The supernatants were transferred to $50 \mathrm{~mL}$ flasks and the volume was adjusted with acidified ethanol. The absorbance of the anthocyanin pigments of the samples was measured at $535 \mathrm{~nm}$ against a reagent blank. The cyanidin 3-glucoside was used as standard for quantifying the total anthocyanin content. A concentration series between 0 and $27 \mu \mathrm{g}$ of cyanidin 3-glucoside was prepared in $3 \mathrm{~mL}$ of acidified ethanol. Total anthocyanin content was expressed on a fresh weight basis.

\section{RNA isolation and Northern blot analysis}

The total RNA was extracted by the hot phenol and lithium chloride precipitation method (Pawlowski et al., 1994). Then, 10 micro grams of total RNA was electrophoretically separated on $1.2 \%(\mathrm{w} / \mathrm{v})$ denaturing formaldehyde agarose gel and blotted onto a nylon membrane (MAGNA Osmonics Inc., Minetonka, MN), following the manufacturer's instructions. After blotting, the RNA was immobilized on the membrane by UV cross-linking $(120 \mathrm{~mJ})$ in a UV Stratalinker (Stratagene, La Jolla, CA, USA). The prehybridization was carried out for 2 to $4 \mathrm{~h}$ at $42^{\circ} \mathrm{C}$ in a solution containing $50 \%(\mathrm{v} / \mathrm{v})$ formamide, 5X Denhardt's solution, $0.1 \%$ (w/v) SDS, 6X SSPE and $150 \mu \mathrm{g} / \mathrm{mL}$ denatured salmon sperm DNA. DNA fragments labeled with ${ }^{32} \mathrm{P}-\mathrm{dCTP}$ using DECA prime $\mathrm{II}^{\mathrm{TM}}$ DNA labeling kit (Ambion, Austin, TX) was used to probe the membranes. Hybridization was carried out with $10^{6} \mathrm{cpm}$ of the gene $A 1$ probe $/ \mathrm{mL}$ at $42^{\circ} \mathrm{C}$ for $16 \mathrm{~h}$ in a fresh prehybridization buffer. The filters were initially washed twice for $10 \mathrm{~min}$ with a low stringency solution consisting of $2 \mathrm{X}$ SSC and $0.2 \%$ SDS (v/v), followed by a high stringency wash with $0.1 \mathrm{X} \mathrm{SSC}$ and $0.1 \% \operatorname{SDS}(\mathrm{v} / \mathrm{v})$ at $42^{\circ} \mathrm{C}$ for $10 \mathrm{~min}$. Membranes were exposed to Kodak XAR-5 films.

\section{RESULTS}

In this study, we evaluated the expression pattern of the maize $A l$ gene coding for dihydroflavonol-4-reductase (DFR) in Pi-efficient and Pi-inefficient genotypes of tropical maize (Table 1). The anthocyanin accumulation in the roots of Pi-efficient and Pi-inefficient genotypes grown for 15 days in hydroponic culture in the presence $(250 \mu \mathrm{M} \mathrm{Pi})$ or absence $(0 \mu \mathrm{M} \mathrm{Pi})$ of phosphate are shown in Figure 1. All genotypes showed an increase in 
anthocyanin accumulation in absence of Pi treatment. The Pi-efficient genotype L-36, and Pi-inefficient genotypes L-16, L22, and L-5046 showed the highest levels of anthocyanin accumulation (Fig. 1).

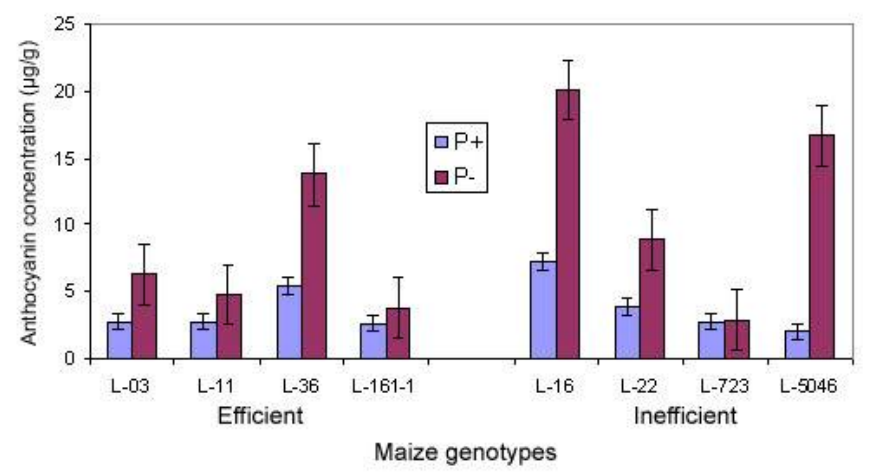

Figure 1. Anthocyanin accumulation in maize roots of Pi-efficient and Pi-inefficient genotypes grown for 15 days in hydroponic culture in the presence $(250 \mu \mathrm{M} \mathrm{Pi})$ or absence $(0 \mu \mathrm{M} \mathrm{Pi})$ of phosphate. The bars represent the mean of three replicates with standard errors.

In response to phosphate deficiency, maize plants showed strong accumulation of the $A 1$ mRNA (Figure 2). Differential expression of the $A l$ gene was observed in both Piefficient and Pi-inefficient genotypes at different concentrations of Pi (data not shown). The differential expression of $A l$ gene in plants of the Pi-efficient (L161-1) and Pi-inefficient (L-5046) genotypes under different Pi concentrations is presented in Figure 3.
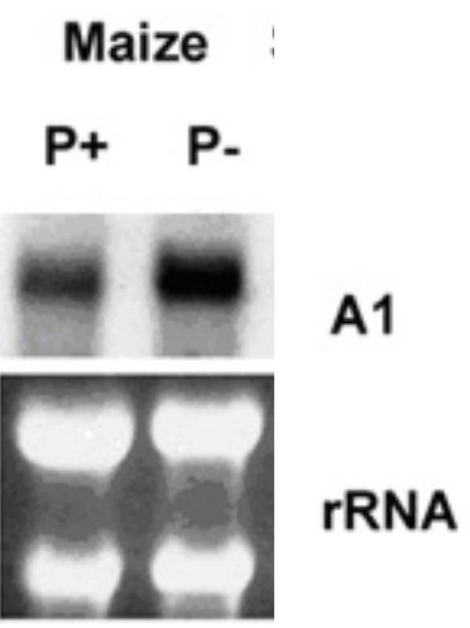

Figure 2. Northern blot of the expression of the $A l$ gene of Pi-efficient and Pi-inefficient maize. Total RNA isolated from the roots of plants grown in hydroponic culture supplied with half-strength modified Hoagland's solution containing $250 \mu \mathrm{M}$ phosphate (+) or no phosphate (-) for 15 days. The blot was probed with ${ }^{32} \mathrm{P}$ labeling. The panel below Northern blots is the ethidium bromide-stained gel prior to blotting to evaluate RNA (10 $\mu \mathrm{g} / \mathrm{lane})$ integrity and uniformity of loading. 


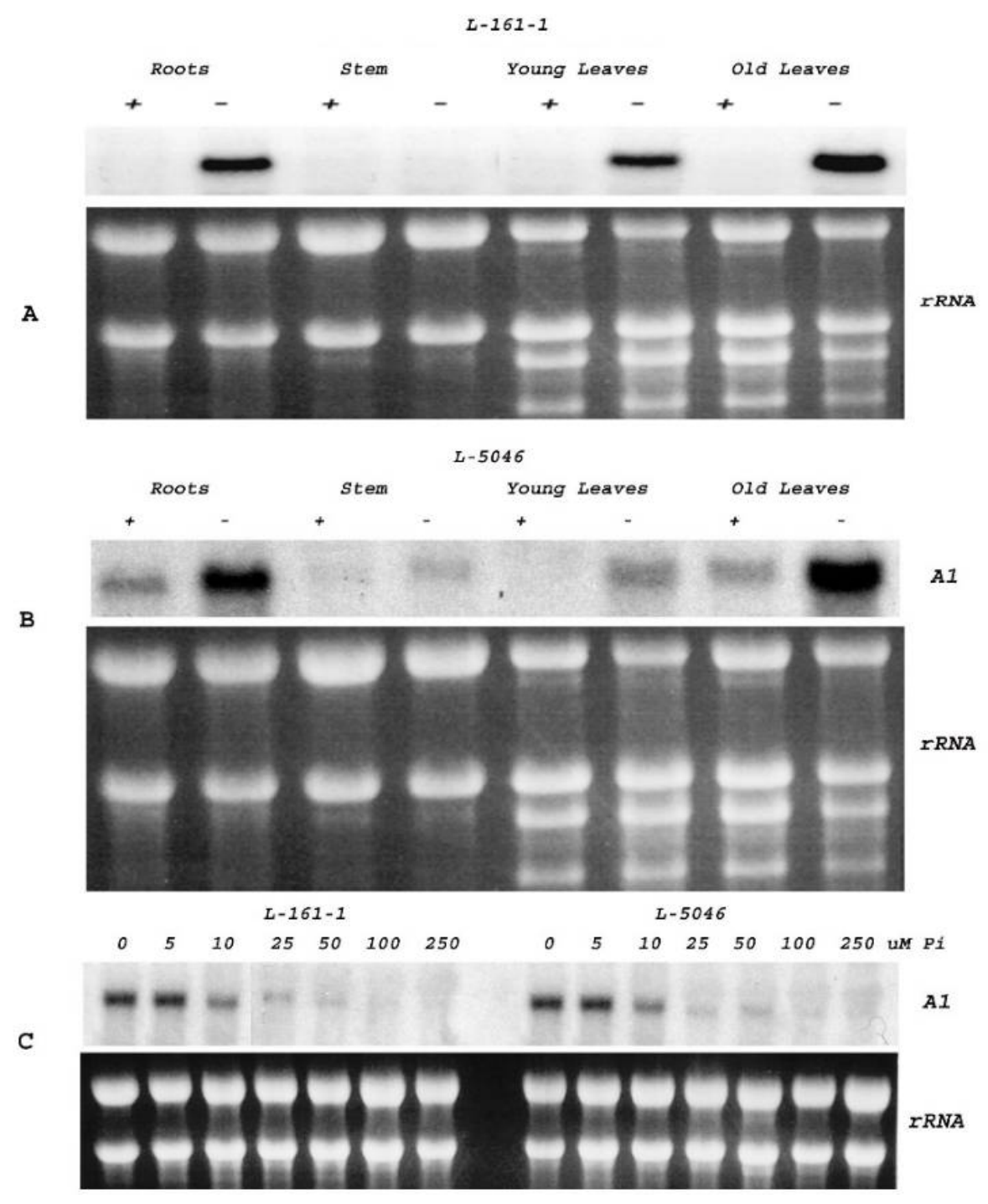

Figure 3. Northern blot analysis of maize $A 1$ gene of Pi-efficient and Pi-inefficient genotypes grown for 15 days in hydroponic culture in the presence or absence of phosphate. A and B show expression of the Al gene in different plant parts of the genotypes L-161-1 (Pi-efficient) and L-5046 (Pi-inefficient) grown under P+ $(250 \mu \mathrm{M}$ $\mathrm{Pi})$ and $\mathrm{P}-(0 \mu \mathrm{M} \mathrm{Pi})$ conditions, and $\mathrm{C}$ shows the expression pattern of the $A 1$ gene in the roots of plants grown in different concentration of Pi. The blots were probed with ${ }^{32} \mathrm{P}$ labeling. The panels below the northern blots are ethidium bromide-stained gel prior to blotting to demonstrate RNA integrity and uniformity of loading.

The $A l$ gene expression in different plant tissues (roots, stems, and young and old leaves) revealed a strong induction in the roots, and young and old leaves of the Pi-efficient genotype (Figure 3A), and in the roots and old leaves of the Pi-inefficient genotype (Figure 3B). The expression pattern of the $A l$ gene in both Pi-efficient and Pi-inefficient genotypes grown in different concentrations of Pi were similar, with the induced expression of the $A I$ 
gene being observed at reduced concentrations of phosphorus ( 0 to $10 \mu \mathrm{M} \mathrm{Pi})$, as shown in Figure 3C. Under Pi concentrations up to $10 \mu \mathrm{M}$, expression of the $A l$ transcripts was barely detectable.

\section{DISCUSSION}

We examined the expression of $A l$ gene in contrasting genotypes of maize in relation to phosphorus efficiency (phosphate efficient/responsive and phosphate inefficient/non-responsive) under phosphorus starvation conditions. We observed quantitative differences in anthocyanin accumulation in maize genotypes grown in the presence or absence of $\mathrm{Pi}$. In the absence of $\mathrm{Pi}$, all maize genotypes (responsive and nonresponsive) showed higher anthocyanin production compared with genotypes grown in the presence of $\mathrm{Pi}$. This result is in accordance with previous studies demonstrating that the accumulation of anthocyanins under Pi starvation is a hallmark of Pi-deficient plants (Chen et al., 2015). The induction of anthocyanin production is part of complex alternative systems developed by plants to adapt to low $\mathrm{Pi}$, including symbiotic interactions with mycorrhizae, the secretion of acid phosphatases, root hair elongation, reduced plant growth, and the up-regulation of phosphate starvation-inducing (PSI) genes (Raghothama, 2000; Nagy et al., 2006; Jain et al., 2007; Hernández and Munné-Bosch, 2015). On the other hand, the addition of phosphate to the suspension culture of Vitis sp. induced cell division and completely inhibited the biosynthesis of anthocyanin (Kakegawa et al., 1995). Collectively known as Pi starvation responses (PSRs), these modifications act in concert to increase Pi uptake from soil and readjust Pi allocation and utilization by the plants (Leong et al., 2018).

The Pi starvation response is mediated by altered expression of a number of genes coding for transcription factors and cell-signaling proteins implying in major regulatory changes in cellular growth and development (Jones et al., 2015; Hoehenwarter et al., 2016; Wege et al., 2016). Among the many Pi starvation responses, accumulation of flavonoids such as anthocyanin in Pi deficient plants is the most noticeable (Jiang et al., 2007). Anthocyanin is synthesized in response to many environmental stimuli, and may induce plant tolerance to a number of biotic and abiotic stresses (Chalker-Scott 1999; Chen et al., 2015). In our study, maize plants accumulated significant amounts of anthocyanin under Pi deficiency conditions.

The production of anthocyanin is controlled by various regulatory and structural genes $(A 1, A 2, C, R$, and $B z)$. The gene $A l$ codes the enzyme dihydroflavonol 4-reductase (DFR), involved in the two branches of the anthocyanin pathway, and recessive mutations of the $A 1$ gene lead to a colorless aleurone layer (Styles and Ceska, 1975). In our study, maize plants showed differential expression of $A l$ gene among Pi-efficient and $\mathrm{Pi}$ inefficient genotypes. Expression of the $A l$ gene was strongly influenced by altered $\mathrm{Pi}$ levels in the media. A strong induction of the $A l$ gene was observed in roots and old leaves of both efficient and inefficient genotypes when the Pi concentration was reduced to $10 \mu \mathrm{M}$. However, the role of the augmented expression of $A l$ gene and anthocyanin accumulation in maize roots is not clear. One possible explanation is that the activation of the anthocyanin pathway may release much-needed Pi for the plants. In the aerial parts of plants, the expression of $A I$ and accumulation of anthocyanin has been associated with a protective effect of the photosynthetic apparatus against photo inhibition (Gould et al., 1995). According to Chen et al. (2015), the accumulation of anthocyanin in leaves of Pi-deficient 
plants serves to protect nucleic acids and chloroplasts against photo-oxidative damage due to limited photosynthesis. However, the adaptive advantages of anthocyanin, in nonreproductive tissues, especially in roots, are much less clear. As reported by Chalker-Scott (1999), the production and localization of anthocyanins in root, stem and especially leaf tissues may allow the plant to develop resistance to a number of environmental stresses. In fact, in our study expression of the $A l$ gene in roots suggests the existence of a different protective mechanism for anthocyanins, beyond their role in photoinhibition, as observed in leaves by Gould et al. (1995). Although further studies are needed to examine the role of anthocyanins in roots of $\mathrm{Pi}$ deficient plants, a positive correlation exists between over expression of the $A l$ gene and anthocyanin accumulation in both Pi-efficient and Piinefficient genotypes grown in absence of Pi. This suggests that the extent of anthocyanin accumulation in the roots may be used as an indicator of P-availability in the soil. Also, our study showed no consistent correlation between phosphate efficiency and Al gene expression or anthocyanin accumulation across the tested genotypes. According to our findings, maize genotypes with varying responses to available phosphate accumulate anthocyanin and activate the $A l$ gene in a phosphate dependent manner. In fact, this became quite evident when two contrasting genotypes (L-161-1 and L-5046) were compared (Fig. 3 ). This leaves the question about utility of anthocyanin accumulation as a marker of phosphate efficiency in tropical maize breeding unanswered. However it will be useful to examine the accumulation of anthocyanin in roots of maize and its potential physiological role under phosphate deficiency.

\section{ACKNOWLEDGMENTS}

The author MJVV give thanks Embrapa, the Brazilian Agricultural Research Corporation, and the McKnight Foundation for financial support.

\section{CONFLICT OF INTEREST}

All authors declare they have no conflicts of interest.

\section{REFERENCES}

Abdel-Aal ESM and Hucl P (1999). A rapid method for quantifying total anthocyanins in blue aleurone and purple pericarp wheats. Cereal Chem. 76: 350-354.

Abreu JBN and Frei M (2016). Microarray meta-analysis focused on the response of genes involved in redox homeostasis to diverse abiotic stresses in rice. Front. Plant Sci. 12: 1260.

Carletti G, Nervo G and Cattivelli L (2014). Flavonoids and melanins: a common strategy across two kingdoms. Int. J. Biol. Sci. 10: 1159-1170.

Chalker-Scott L (1999). Environmental significance of anthocyanin in plant stress response. Photochem. Photobiol. 70: $1-9$.

Chen CY, Wu K and Schmidt W (2015). The histone deacetylase HDA19 controls root cell elongation and modulates a subset of phosphate starvation responses in Arabidopsis. Sci. Rep. 5: 15708.

Edwards CL, Maguire RO, Alley MM, Thomason WE, et al. (2016). Plant-available phosphorus after application of synthetic chelating agents. Commun. Soil Sci. Plant Anal. 47: 433-446.

Fageria NK, Morais OP, dos Santos AB and Vasconcelos MJ (2014). Phosphorus use efficiency in upland rice genotypes under field conditions. J. Plant Nutr. 37: 633-642.

Glover BJ and Martin C (2012). Anthocyanins. Current Biol. 22: R147-R150.

Gould KS, Kuhn DN, Lee DW and Oberbauer SF (1995). Why leaves are sometimes red. Nature 378: 241-242.

Gu M, Chen A, Sun S and Xu G (2016). Complex regulation of plant phosphate transporters and the gap between molecular mechanisms and practical application: what is missing? Mol. Plant. 9: 396-416. 
Hasan M, Hasan M, Silva JAT and Li X (2016). Regulation of phosphorus uptake and utilization: transitioning from current knowledge to practical strategies. Cellular Mol. Biol. Letters. 21: 1-19.

Hernández I and Munné-Bosch S (2015). Linking phosphorus availability with photo-oxidative stress in plants. J. Exp. Bot. 66: 2889-2900.

Hoehenwarter W, Mönchgesang S, Neumann S, Majovsky P, et al. (2016). Comparative expression profiling reveals a role of the root apoplast in local phosphate response. BMC Plant Biol. 16: 106-127.

Jain A, Vasconcelos MJV, Sahi S and Raghothama KG (2007). Molecular mechanisms of plant adaptation to phosphate deficiency. Plant Breed. Rev. 29: 359-419.

Jones C, Hatier, JH, Cao M, Fraser K, et al. (2015). Metabolomics of plant phosphorus-starvation response. In: Annuals Plant Reviews: Phosphorus Metabolism in Plants, (Plaxton WC, Lambers H (eds.). John Wiley \& Sons, Oxford, 217-231.

Kakegawa K, Suda J, Sugiyama M and Komamine A (1995). Regulation of anthocyanin biosynthesis in in relation to cell division. Physiol. Plant. 94: 661-666.

Mehra P, Pandey BK and Giri J (2015). Comparative morphophysiological analyses and molecular profiling reveal Piefficient strategies of a traditional rice genotype. Front. Plant Sci. 6: 1184.

Młodzińska E and Zboińska M (2016). Phosphate uptake and allocation - A closer look at Arabidopsis thaliana L. and Oryza sativa L. Front. Plant Sci. 7: 1198.

Muchhal US and Raghothama KG (1999). Transcriptional regulation of plant phosphate transporters. Proc. Natl. Acad. Sci. 96: 5868-5872.

Nagy $R$ Vasconcelos MJV, Zhao S, McElver J, et al. (2006). Differential regulation of five pht1 phosphate transporters from maize (Zea mays L.). Plant Biol. 8: 186-197.

Pawlowski K, Kunze R, De Vries S and Bisseling T (1994). Isolation of total, poly(A) and polysomal RNA from plant tissues. In: Plant Molecular Biology Manual (Gelvin SB and Schilperoort RA, eds.). The Netherlands: Kluwer Academic Publishers, Dordrecht, 231-243.

Poirier Y and Bucher M (2002). Phosphate transport and homeostasis in Arabidopsis. The Arabidopsis Book 1:e0024. American Society of Plant Biologists, Rockville. Available at [https://doi.org/10.1199/tab.0024]. Accessed January $18,2018$.

Puga MI, Rojas-Triana M, Lorenzo L, Leyva A, et al. (2017). Novel signals in the regulation of Pi starvation responses in plants: facts and promises. Current Opinion in Plant Biol. 39: 40-49.

Raghothama KG and Karthikeyan AS (2005). Phosphate acquisition. Plant Soil. 274: 37-349.

Raghotama KG (2000). Phosphate transport and signaling. Curr. Opin. Plant Biol. 3: 182-187.

Raghothama KG (1999). Phosphate acquisition. Annu. Rev. Plant Physiol. Plant Mol. Biol. 50: 665-693.

Schachtman DP, Reid RJ and Ayling SM (1998). Phosphorus uptake by plants: from soil to cell. Plant Physiol. 116: 447-453.

Styles ED and Ceska O (1975). Genetic control of 3-Hydroxy-and 3-deoxy-flavonoids in Zea mays. Phytochem. 14: 413 415.

Wege S, Khan GA, Jung JY, Vogiatzaki E, et al. (2016). The EXS domain of PHO1 participates in the response of shoots to phosphate deficiency via a root-to-shoot signal. Plant Physiol. 170: 385-400.

Zhang Z, Liao H and Lucas WJ (2014). Molecular mechanisms underlying phosphate sensing, signaling, and adaptation in plants. J. Integr. Plant Biol. 56: 192-220. 